\title{
Analysis of Temperature Field in Auto Starter Based on FEM
}

\author{
Jun $\mathrm{Li}^{1, \mathrm{a}}$, Hailong Deng ${ }^{1, \mathrm{~b}}$ and Pengfei Huang ${ }^{1, \mathrm{c}}$ \\ 1 East China Jiaotong University, Nanchang, China \\ a65770426@qq.com, b1357606432@qq.com, ${ }^{\mathrm{c}} 786556915 @ q q . c o m$
}

Keywords: auto starter, temperature field, FEM.

Abstract. Starter motor is an important part of the auto starting system. The analysis method of temperature field for auto starter based on FEM was given. The size of heat source and the heat transfer coefficient of materials were determined. Taking 1/4 model of motor as the research object, the finite element grid could be obtained after setting the simulation boundary conditions. So the steady-state temperature field of the starter was calculated. The calculation results show that the temperatures of the excitation winding and the rotor winding in the starter is higher and it is the main causes of burn during the using of the starter. The method given in the paper can provide guidance to improve the structural parameters of the starter.

\section{Introduction}

As people increasingly concerned about environment and resource consumption issues, start-stop system as a car environmental protection technology has been popularized rapidly ${ }^{[1]}$. At the same time, the surge of start-stop frequency put forward higher requirements on the starter heat resistance. Winding coil burning is one of problems of the starter in the car driving process. Before dealing with such issues effectively, the starter temperature field needs to be analyzed, according to the heat distribution on starter to study its thermal carrying capacity based on the steady state.

\section{Modeling}

The actual structure of the starter is relatively complicated. In order to facilitate the calculation, some simplifications and assumptions are needed: 1)Motor impregnated paint in good condition and filled evenly; 2) Insulation paint on copper wire distributed evenly; 3) Due to the multi-layer sheet structure of silicon steel lead to the thermal conductivity of the core in the axial and radial differ greatly, this paper only consider the radial thermal conductivity; 4)The geometric position of excitation winding and rotor winding is the ideal position, ignoring the installation error.

Simulation calculation for a starter motor product, the relevant parameters is shown in Table 1.

Table 1 Part of the parameters on starter motor

\begin{tabular}{cc||cc}
\hline Parameter name & Value & Parameter name & Value \\
\hline Chassis $/ \mathrm{mm}$ & 0.8 & Rotor wire $/ \mathrm{mm}$ & 0.5 \\
Magnetic pole/mm & 0.8 & Insulating paint of slot $/ \mathrm{mm}$ & 0.3 \\
Air part $/ \mathrm{mm}$ & 0.3 & Rotor shaft $/ \mathrm{mm}$ & 0.5 \\
Excitation winding $/ \mathrm{mm}$ & 0.5 & Rotor core $/ \mathrm{mm}$ & 0.5 \\
\hline
\end{tabular}

Maxwell 2D software was used to establish a 1/4 two-dimensional model, and after mesh partition, the finite element model is shown in Figure 1. The boundary conditions include wall and symmetrical boundaries, filled air in the area between the stator and the rotor, in contact with the field winding, rotor, pole and stator housing. 


\section{Solve the parameters}

Equivalent thermal conductivity of air gap. The effective thermal conductivity of stationary fluid is used to describe the heat exchange capacity of the flowing air in the air gap. That is to say, the heat transferred by the stationary fluid between the stationary rotors per unit time is equal to the heat transmitted by the flowing air, Rotating rotor can be regarded as stationary ${ }^{[2]}$. After analysis and calculation, the Air flow in the air gap is laminar flow, the effective thermal conductivity is equal to the thermal conductivity of air.

The thermal conductivity of rotor core. Since the rotor is stacked with silicon lamination, air is left between the steel sheets and the core lamination and the motor windings are impregnated with paint, so the heat transfer coefficient is much smaller than the thermal conductivity of the silicon lamination, so the equivalent thermal conductivity can be used to simulate the actual situation. The equivalent thermal conductivity of rotor is calculated as follows:

$$
\lambda=\frac{\delta_{F e}+\delta_{0}}{\frac{\delta_{F e}}{\lambda_{1}}+\frac{\delta_{0}}{\lambda_{0}}}=\frac{1}{\frac{K_{F e}}{\lambda_{1}}+\frac{1-K_{F e}}{\lambda_{0}}}
$$

Where $K_{F e}$ is stack coefficient of rotor core; $\lambda_{1}$ is the thermal conductivity of silicon lamination in rotor core; $\lambda_{0}$ is the thermal conductivity of insulating paint (it is generally $5 \sim 10$ times the air).

The equivalent thermal conductivity of winding. Stator windings and rotor windings are surrounded by insulator, they are impregnated with insulating paint and the material is complex, so it also uses the equivalent thermal conductivity to describe:

$$
\begin{aligned}
& K_{s}=\frac{1}{R_{s}} \\
& R_{s}=\frac{\delta_{i}}{\lambda_{i}}+\frac{1}{4}\left[\frac{b\left(1-\sqrt{S_{f}}\right)}{\lambda_{L}} K_{L}+\frac{b\left(1-\sqrt{S_{f}}\right)}{\lambda_{a}}\left(1-K_{L}\right)+\frac{d-d_{W}}{\lambda_{d}} \frac{b \sqrt{S_{f}}}{d}\right]
\end{aligned}
$$

Where $\delta_{i}$ is thickness of slot insulation; $\lambda_{l}$ is the thermal conductivity of slot insulation, polyester film is $0.15 \mathrm{~W} /(\mathrm{mUK}) ; \lambda_{L}$ is the thermal conductivity of varnish, setting $0.17 \sim 0.18 \mathrm{~W} /(\mathrm{m} \hat{K}) ; \lambda_{d}$ is the thermal conductivity of paint layer on wire, the polyester enameled wire is $0.15 \mathrm{~W} /(\mathrm{mûK}) ; S_{f}$ is the full rate of slot; b is the width of slot, $b=2 R_{S^{-}}\left(2 R_{s^{-}} b_{S I}\right) 1 / 3 ; K_{L}$ is Filling coefficient of paint, setting $0.85 \mathrm{~W} /(\mathrm{m} \hat{\mathrm{K}})$; $d$ is outside diameter of enameled wire, $d_{W}$ is outside diameter of bare wire.

The thermal conductivity of slot insulation. The rotor slot is filled with varnish, and the thermal conductivity of the varnish is $0.14 \sim 0.16 \mathrm{~W} /(\mathrm{mûK}){ }^{[3]}$.

The heat dissipation coefficient of chassis. Because there is a gas circulation in the chassis, the following formula can be used to calculate the heat dissipation coefficient of chassis ${ }^{[4]}$ :

$$
a=14\left(1+0.5 \sqrt{\omega_{i}}\right) \sqrt[3]{\frac{\theta}{25}}
$$

Where $a$ is heat transfer coefficient on surface, $\mathrm{W} /\left(\mathrm{m}^{2} \hat{\mathrm{UK}}\right) ; \omega_{i}$ is the wind speed inside the engine base, $\mathrm{m} / \mathrm{s} ; \theta$ is temperature on the outer surface of the engine base, $\mathrm{K}$. Taking the relative speed of the rotor circumference and the magnetic pole as a reference, the heat dissipation coefficient of chassis can be calculated as $30.783 \mathrm{~W} /\left(\mathrm{m}^{2} \mathrm{U}\right.$ K).

The thermal conductivity of the other components for the above simulation is described as follows: 
Table 2 Thermal conductivity of each component

\begin{tabular}{cc||cc}
\hline Part name & $\begin{array}{c}\text { Thermal Conductivity } \\
\left(\mathrm{Wu}\left(\mathrm{m}_{\mathfrak{l}}\right)^{-1}\right)\end{array}$ & Part name & $\begin{array}{c}\text { Thermal Conductivity| } \\
\left(\mathrm{W} \hat{\mathrm{u}}\left(\mathrm{m}_{\mathfrak{l}} \mathrm{k}\right)^{-1}\right)\end{array}$ \\
\hline Magnetic pole & 40 & Rotor / field winding & 110 \\
Rotor core & 35.6 & Chassis & 40 \\
Impregnated paint of Rotor & 0.16 & Air layer & 0.003 \\
\hline
\end{tabular}

The core loss mainly exists in the stator part, and the stator iron loss is loaded on the stator shell; the excitation copper loss is loaded on the excitation coil; and the rotor copper loss is loaded on the rotor wire. Since the iron loss created by the magnetic field changes on the case and pole surfaces is transmitted directly on the ANSYS Workbench ${ }^{[5]}$ platform, all the heat sources in the motor are obtained by adding copper losses on the motor field coils and the rotor wires. The excitation winding copper consumption value is $0.622 \mathrm{~kW}$, copper rotor winding value is $0.782 \mathrm{kw}$ in the process of calculating.

Since the model of the calculation motor is a 1/4 dimensional model, the power of each part of the heat source can not be directly loaded. Therefore, it is necessary to calculate the volume of the copper wire used for the winding, calculate the heat generating rate of per unit volume of the copper wire by using the winding loss power and the copper wire volume, enter the heat rate value in Fluent. The heat source power is converted into the heat generation rate of a single wire in a two-dimensional model: the heat generation rate of the field winding is $2.212 \times 10^{7} \mathrm{~W} / \mathrm{m}^{3}$ and the heat generation rate of the rotor winding is $2.144 \times 10^{6} \mathrm{~W} / \mathrm{m}^{3}$.

\section{Simulation results and analysis}

Before calculating the temperature field of the starter, the following assumptions are made: 1) the density, specific heat capacity, and thermal conductivity of all materials do not vary with time and temperature; 2) the copper loss is evenly distributed in the windings; 3) the ambient temperature does not vary with time, setting it as 40 centigrade.

The model will be calculated in Fluent temperature, the temperature distribution of the cloud chart is shown in Figure 2. Figure 2 shows the maximum temperature of the starter is up to $121^{\circ} \mathrm{C}$, located in the stator field winding; the lowest temperature is at the outer surface of the case, it's $52^{\circ} \mathrm{C}$. As the excitation winding copper consumption, the rotor winding copper consumption and iron core stator iron core are the main heat source, the heat generated by the winding conduction in turn to its neighboring media and chassis, and finally dissipated into the air by the chassis, the simulation result is in line with the actual situation.

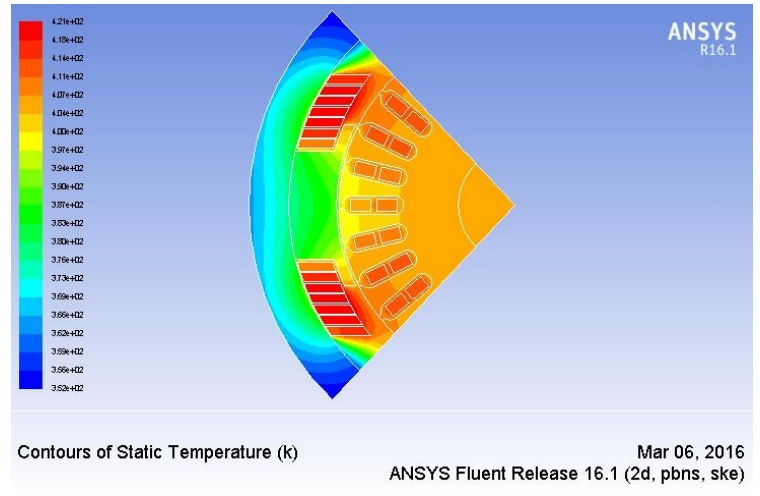

Fig.2 Temperature distribution of model

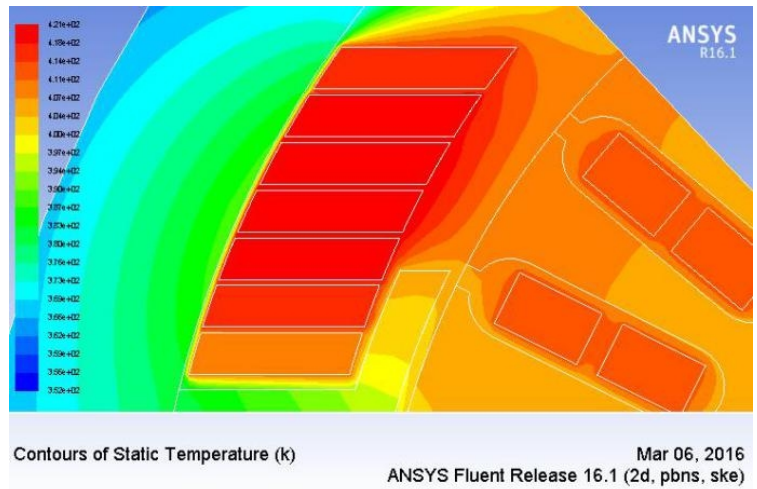

Fig.3 Temperature distribution 
Excitation winding local temperature cloud is shown in Figure 3. As can be seen in Figure 3, the overall temperature field winding is distributed evenly, the maximum temperature is $121^{\circ} \mathrm{C}$, the lowest temperature is $110^{\circ} \mathrm{C}$, nearing the magnetic pole. The reason is that the thermal conductivity of the magnetic pole is higher compared with the air, insulating material, and the thermal conductivity of copper in the winding material is good, thus the temperature range of the winding is smaller.

The temperature distribution of the rotor windings is symmetrical, this paper only intercepts the upper part, as shown in Figure 4. The maximum temperature of motor rotor winding is $112^{\circ} \mathrm{C}$, the minimum temperature is $105^{\circ} \mathrm{C}$. The overall temperature is lower than the excitation winding, which is due to the distribution of the rotor winding is relatively dispersed, distributed in the rotor core of the 27 wire slots, the heat can be dispersed to the rotor core; In addition, the rotor winding wire slot in the rotor is impregnated with paint immersion, varnish and wire impregnation, varnish and rotor core completely fit, easy to dissipate heat.

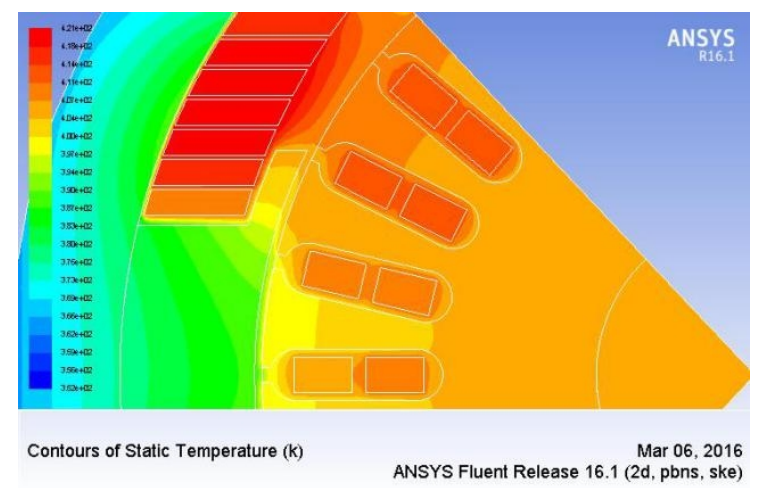

Fig.4 Temperature distribution of the rotor windings

\section{Conclusion}

This paper presents the finite element method of starter temperature field, determines the size of heat source and the heat transfer coefficient of each material, calculates and analyzes the steady temperature field of a starter, therefore the simulation results are true and effective. The calculation results show that the temperature of the excitation winding and the rotor winding in the starter are both high. Although there is input of iron loss heat source in the magnetic pole and the shell, the heat dissipation condition is good, So that it won't led to heat concentration. Excitation winding temperature is relative higher than the rotor winding temperature, mainly because heat source concentration and cooling conditions is relatively poor which is the main reason for the starter burning. Finally, the calculation method given in this paper is of theoretical guidance to improve the structure and parameters set of the starter.

\section{Acknowledgements}

This work was financially supported by the Key R\&D Project of Jiangxi Provincial Department of Science and Technology (20161BBE50078).

\section{References}

[1] Li Min, Xi Chung min, Li Zhekun, He Kaixin, Dong Songmei. Study on smart start system of Automobile [J]. Journal of changshu institute of technology, 2013,02:79-83.

[2] Huang Dongzhu. Large and medium-sized asynchronous motor electromagnetic field and temperature field numerical calculation [D]. Harbin University of Science and Technology, 2004.

[3] Wei Yongtian, Meng Dawei, Wen Jiabin. Motor heat exchange [M]. Beijing: Mechanical Industry Press, 1998. 
[4] Han Li, Jiao Xiaoyan, Li Jingcan, Wang Hua. Analysis and calculation of the whole range temperature field of the brushless doubly fed machine, [J]. Journal of motor and control, 2013, 17(5):1-8.

[5] Huang Zhixin, Liu Chengzhu. Super learning handbook of ANSYS Workbench 14.0 [M]. Beijing: The press of people's post and telecommunications university, 2013. 